\title{
Recombinant TIMP-1-GPI inhibits growth of fibrosarcoma and enhances tumor sensitivity to doxorubicin
}

\author{
Q. Bao $\cdot$ H. Niess $\cdot$ R. Djafarzadeh $\cdot$ Y. Zhao $\cdot$ B. Schwarz $\cdot$ \\ M. K. Angele • K.-W. Jauch • P. J. Nelson • C. J. Bruns
}

Received: 21 March 2013 / Accepted: 26 July 2013 / Published online: 10 August 2013

(C) The Author(s) 2013. This article is published with open access at Springerlink.com

\begin{abstract}
Fibrosarcomas show a high incidence of recurrence and general resistance to apoptosis. Limiting tumor regrowth and increasing their sensitivity to chemotherapy and apoptosis represent key issues in developing more effective treatments of these tumors. Tissue inhibitor of metalloproteinase 1 (TIMP-1) broadly blocks matrix metalloproteinase (MMP) activity and can moderate tumor growth and metastasis. We previously described generation of a recombinant fusion protein linking TIMP-1 to glycosylphophatidylinositol (GPI) anchor (TIMP-1-GPI) that efficiently directs the inhibitor to cell surfaces. In the present report, we examined the effect of TIMP-1-GPI treatment on fibrosarcoma biology. Exogenously applied TIMP-1-GPI efficiently incorporated into surface membranes of human HT1080 fibrosarcoma cells. It inhibited their proliferation, migration, suppressed cancer cell clone formation, and enhanced apoptosis. Doxorubicin, the standard chemotherapeutic drug for fibrosarcoma, was tested alone or in combination with TIMP-1-GPI. In parallel, the influence of treatment on HT1080 side population cells (exhibiting tumor
\end{abstract}

Q. Bao and H. Niess have contributed equally to this manuscript.

Electronic supplementary material The online version of this article (doi:10.1007/s11523-013-0294-5) contains supplementary material, which is available to authorized users.

\section{Q. Bao}

Department of Plastic and Reconstructive Surgery, 2nd Affiliated Hospital, School of Medicine, Zhejiang University, Hangzhou,

Zhejiang, China

Q. Bao $\cdot$ H. Niess $\cdot$ Y. Zhao $\cdot$ B. Schwarz $\cdot$ M. K. Angele $\cdot$

K.-W. Jauch $\cdot$ C. J. Bruns

Department of Surgery, University of Munich, Campus Grosshadern, Munich, Germany

R. Djafarzadeh • P. J. Nelson ( $\bowtie)$

Clinical Biochemistry Group, Medizinische Klinik und Poliklinik IV,

University of Munich, Schillerstrasse 42,

D-80336 Munich, Germany

e-mail: peter.nelson@med.uni-muenchen.de stem cell-like characteristics) was investigated using Hoechst 33342 staining. The sequential combination of TIMP-1-GPI and doxorubicin showed more than additive effects on apoptosis, while TIMP-1-GPI treatment alone effectively decreased "stem-cell like" side population cells of HT1080. TIMP-1-GPI treatment was validated using HT1080 fibrosarcoma murine xenografts. Growing tumors treated with repeated local injections of TIMP-1-GPI showed dramatically inhibited fibrosarcoma growth and reduced angiogenesis. Intraoperative peritumoral application of GPI-anchored TIMP-1 as an adjuvant to surgery may help maintain tumor control by targeting microscopic residual fibrosarcoma cells and increasing their sensitivity to chemotherapy

Keywords Tissue inhibitors of metalloproteinases (TIMPs) GPI-anchor · Fibrosarcoma · Chemo-sensitivity ·

Cancer stem cells

\section{Introduction}

Tumor progression is dependent on interactions between the tumor and the surrounding stromal tissue. These processes are mediated in part by a complex system of proteases and inhibitors including the family of matrix metalloproteinases (MMPs), and their natural inhibitors, the family of tissue inhibitors of matrix metalloproteinases (TIMPs). The extracellular matrix (ECM) contains a pool of cytokines, growth factors and additional components with unknown function. MMPs not only promote spatial invasion of tumor cells by degradation of the ECM, but they also help to process products and growth factors that have important signaling functions for gene expression and cell behavior [1]. In this way, a complex network of proteases/inhibitors is associated with the invasive front of a progressing cancer.

Inhibition of MMPs has long been proposed as a potential treatment option for cancers. However, despite promising pre- 
clinical studies, clinical trials with broad-spectrum inhibitors of MMPs have failed to demonstrate effectiveness [2]. The lack of efficacy shown with systemic MMP inhibition is in part linked to the complexity of protease networks. This socalled protease spam may affect tumor growth locally in a paracrine fashion and have systemic/endocrine effects [3]. Therefore, more specific and selective strategies of MMP inhibition need to be developed.

TIMP-1 is a soluble protein that can be occasionally detected on the cell surface through its interaction with surface proteins and is a natural inhibitor of most MMPs [4-14]. TIMP-1 was originally identified as a naturally occurring intrinsic barrier to tumor invasion $[15,16]$. However, studies on cancer proliferation and growth have shown both pro- and anti-tumorigenic effects for TIMP-1 depending on the tumor and concentration of protein expressed [17-22].

Protein engineering of cell surfaces represents a powerful tool to study and to expand protein function [23]. Proteins anchored by glycophosphatidylinositol (GPI), when purified and added to cells in vitro, are efficiently reincorporated into their surface membranes [23-26]. Thus, a fusion protein of TIMP-1-GPI allows the efficient localization of exogenously added TIMP-1 selectively on to cell membranes independent of protein-protein interactions [23-26]. This has been previously shown to result in significant changes in protease stoichiometry with concomitant alterations in the biological behavior of cells [26]. These effects include enhanced wound healing in some instances, and a general inhibition of tumor growth [27].

Recent studies have suggested that a subpopulation of cancer cells with distinct stem cell-like properties may be largely responsible for cancer initiation, invasion and metastasis formation. This subpopulation has been referred to as cancer stem cells (CSCs) [28, 29]. These cells can efflux chemotherapy drugs, and may thus also account for resistance of tumors to chemotherapy. CSCs can be defined and isolated by different methods, including through the expression of CSC-specific cell surface markers, sphere growth, aldehyde dehydrogenase (ALDH) activity assays, and by the sorting of tumor cell side populations detected by exclusion of Hoeschst 33342 dye. Parallel to what is found in ex vivo tumor samples, side population (SP) cells can also be identified in some tumor cell lines. These SP cells can also exhibit cancer stem cell-like characteristics. Alterations in regulatory pathways and gene networks support the suggestion that SP cells resemble CSCs and may thus effectively model cell types responsible for tumor initiation, relapse, metastasis and chemoresistance $[28,29]$. Recently, the importance of these cells in sarcoma, a tumor entity with high rates of relapse and chemoresistance, was demonstrated [30].

In the present study, we assessed the effects of exogenously added TIMP-1-GPI on the biology of fibrosarcoma, including its influence on the SP population formation and sensitivity to chemotherapy.

\section{Materials and methods}

Ethics statement

The research meets all applicable standards for the ethics of experimentation and research integrity. The animal studies were approved by and conducted in accordance with the principles of the regulatory agency of the State of Bavaria, Germany (Tierversuchsantragsnummer is Gz.: 55.2-1-54-2532-122-09).

Cell lines and culture medium

HT1080, a human fibrosarcoma cell line [31], was kept in DMEM cell culture medium (DMEM low glucose, GIBCO BRL, Life Technologies GmbH, Eggenstein, Germany) supplemented with $10 \%$ fetal bovine serum (FBS, Biochrom AG, Berlin, Germany), 100 units/ml penicillin, and $100 \mu \mathrm{g} / \mathrm{ml}$ streptomycin (PAN biotech $\mathrm{GmbH}$, Aidenbach, Germany) and incubated at $37{ }^{\circ} \mathrm{C}$ under $5 \% \mathrm{CO}_{2}$. Cells were passaged by brief trypsination with $0.025 \%$ trypsin (Trypsin/EDTA, PAN biotech $\mathrm{GmbH}$, Aidenbach, Germany). The cell line was routinely checked and found to be free of mycoplasma contamination.

\section{Purification of TIMP-1-GPI protein}

The TIMP-1-GPI protein was produced and purified as previously described [25]. Briefly, human TIMP-1 was cloned from cDNA using hTIMP-1-specific primers, fused without a translation stop codon to the GPI-signal sequence cloned from LFA-3 $[23,32]$ and subcloned into pEF-DHFR. The expression plasmid was stably introduced into DHFR-deficient Chinese hamster ovary $(\mathrm{CHO})$ cells and selected as described [33]. TIMP-1-GPI fusion protein was purified from the CHO cells by Triton X-100 detergent extraction, followed by column purification using DEAE, heparin sepharose and size exclusion [25].

Incorporation of recombinant TIMP-1-GPI into cell membranes

HT1080 cells $\left(10^{6}\right.$ cells $\left./ \mathrm{ml}\right)$ were incubated with 7 or $14 \mathrm{ng} / \mathrm{ml}$ of purified TIMP-1-GPI or equivalent recombinant human TIMP-1 (rhTIMP-1) for $2 \mathrm{~h}$ at $37^{\circ} \mathrm{C}$. The cells were then washed three times with cold PBS and analyzed by flow cytometry, clone formation, or migration assays.

Fluorescent staining

Surface-associated TIMP-1 protein was detected using human specific TIMP-1 monoclonal antibody. Incorporated cells were detached with Trypsin/EDTA and seeded 2500 cells/ well in duplicate Chamber Slide (8-Well Glass Slide, Lab- 
Tek, NUNC, Rochester, NY, USA). After 24 h, the cells were fixed with $4 \%$ paraformaldehyde for $15 \mathrm{~min}$ in room temperature, After PBS washing and Donkey-serum blocking, the cells were incubated with TIMP-1 monoclonal antibody (IM32, Anti-TIMP-1 Mouse mAb, Calbiochem, Japan) overnight in $4{ }^{\circ} \mathrm{C}$. The cells were then washed three times with PBS, incubated with Goat polyclonal secondary antibody to mouse IgG-H\&L FITC (Abcam, USA), and the nuclei were stained with DAPI. The slides were observed under fluorescent microscope.

\section{Fluorescence-activated cell sorting analysis}

Cells were detached with $0.025 \%$ trypsin (Trypsin/EDTA, PAN biotech $\mathrm{GmbH}$, Aidenbach, Germany) and were kept in dark and cold blocked with FcR blocking reagent (Miltenyi Biotec GmbH, Bergisch Gladbach, Germany) $15 \mathrm{~min}$, and incubated for $45 \mathrm{~min}$ on ice with antibodies specific for human TIMP-1(IM32, Anti-TIMP-1 Mouse mAb, Calbiochem, Japan). Cells were washed three times with $1 \times \mathrm{PBS}$, incubated with FITC-conjugated donkey anti-mouse mAB (Abcam, USA) for $45 \mathrm{~min}$ at $4{ }^{\circ} \mathrm{C}$ and Mouse IgG1-FITC (Miltenyi Biotec $\mathrm{GmbH}$, Bergisch Gladbach, Germany) as negative control, then washed three times with $1 \times$ PBS and analyzed using a flow cytometer (FACSCalibur, Becton Dickinson and Company, San Jose, CA, USA) and CellQuest software.

\section{Viability/proliferation assay}

The MTT colorimetric assay was used to measure cellular metabolic activity via NAD(P)H-dependent cellular oxidoreductase enzymes which are linked to cellular viability and proliferation. HT1080 cells $\left(1.0 \times 10^{4}\right)$ in $200 \mu$ medium were cultured in 96-well plates for $24 \mathrm{~h}$ under standard conditions to yield firmly attached and stably growing cells. After discarding supernatants, $50 \mu \mathrm{l}$ of various concentrations of TIMP-1-GPI (2, 4, 6, 8, 10, 12 and $14 \mathrm{ng} / \mathrm{ml})$, rhTIMP $(14 \mathrm{ng} / \mathrm{ml})$ or vehicle were added and the cells were incubated for 2 or $4 \mathrm{~h}$. The supernatants were then discarded, and the cells were cultured with serum-free medium for another $24 \mathrm{~h}$. Then $100 \mu \mathrm{l}$ of a $10 \%$ solution of Cell counting kit-8 (CK0413, Dojinbo, Kumamoto, Japan) was added. After $2 \mathrm{~h}$ incubation at $37{ }^{\circ} \mathrm{C}$, absorbance was measured at $450 \mathrm{~nm}$ using VersaMax Microplate Reader (Molecular Devices, Sunnyvale, CA, USA). For each experiment, at least six wells were analyzed per experimental condition.

\section{Clonogenic assay}

The ability of tumor cells to form colonies was assessed using the clonogenic assay. After incorporation with TIMP-1-GPI, rhTIMP-1 or vehicle, the HT1080 cells were harvested by trypsinization and seeded as triplicates in 6-well plates at 200 cells per well and allowed to form colonies in an undisturbed, humidified, $37{ }^{\circ} \mathrm{C} / 5 \% \mathrm{CO}_{2}$ air atmosphere. After 10 days, the plates were washed with $1 \times$ PBS and cell colonies were stained with crystal violet solution (Sigma-Aldrich, Steinheim, Germany). Only colonies containing at least 50 cells were considered to be viable survivors.

\section{Migration assay}

After incorporation with TIMP-1-GPI, HT1080 cells were cultured in serum-free medium $24 \mathrm{~h}$ before the start of the migration assay. The cells were detached, and $2.5 \times 10^{4}$ cells/ well cells were seeded to the upper chamber of the migration assay set (Cell Culture Insert $8.0 \mu \mathrm{m}$, BD Falcon, NJ, USA). The bottom chamber was filled with $10 \%$ FBS medium. After $6 \mathrm{~h}$ of incubation, the cells on the upper surface of the filters were completely removed by wiping with a cotton swab, as monitored visually under high magnification. The chambers were washed 3 times with PBS and fixed with $4 \%$ Paraformaldehyde, then detected by $1 \mathrm{mg} / \mathrm{ml}$ crystal violet dye. Cells that had transmigrated the lower surface of the chambers were counted under a microscope at a magnification of $\times 200$. The migrated cell number was determined by three randomized fields of high magnification.

\section{Apoptosis assay}

After treatment with TIMP-1-GPI or rhTIMP, the cells were pelleted and resuspended in staining solution (Annexin-V-FITClabeling reagent and PI in PBS buffer) for $15 \mathrm{~min}$ at room temperature as manufacturer suggested (Annexin-V-FITC kit, MACS Miltenyi Biotec, Bergisch Gladbach, Germany). The cells were then analyzed by flow cytometry (BD FACSCalibur, Becton Dickinson and Company, New Jersey, USA) and CellQuest software. Doxorubicin hydrochloride (D151510MG, Sigma-Aldrich, USA) was added at a concentration of $1 \mathrm{ng} / \mathrm{ml}$ to the fibrosarcoma cell line HT1080 for $24 \mathrm{~h}$.

Flow cytometry analysis for side population cells

The ability to discriminate SP cells is based on the differential efflux of Hoechst 33342 by multi-drug-like ATP-binding cassette (ABC) transporters. Single-cell suspensions of HT1080 untreated or treated for $2 \mathrm{~h}$ with TIMP-1-GPI at $14 \mathrm{ng} / \mathrm{mL}$ were stained with $2.5 \mu \mathrm{g} / \mathrm{ml}$ Hoechst 33342 (Sigma-Aldrich $\mathrm{GmbH}$, Steinheim, Germany) for $60 \mathrm{~min}$ at $37{ }^{\circ} \mathrm{C}$. 225uM Verapamil hydrochloride (Sigma-Aldrich $\mathrm{GmbH}$, Steinheim, Germany) was used to block the ABC transporters as control. Propidium iodide $(10 \mu \mathrm{g} / \mathrm{ml})$ was used to discriminate viable and nonviable cells. Cells were analyzed with a LSR II flow cytometer (BD, Heidelberg, Germany) equipped with 20-mW, 355 nm UV laser. Hoechst 
blue fluorescence was measured with a 450/50BP and Hoechst red with a 670/30BP filter.

In vivo tumor model

Female 6-10-week-old BALB/c nu/nu mice were purchased from Charles River Inc. (Sulzfeld, Germany). Animals were exposed to 12-h-light/12-h- darkness cycles, and standard food and water was provided on request. All of the animal studies were approved by and conducted in accordance with the principles of the regulatory agency of the State of Bavaria. HT1080 cells at a concentration of $1.0 \times 10^{6} / 200 \mu$ l cells were implanted subcutaneously at the upper dorsal aspect of the vertebral column under general anesthesia, and the growth characteristics of the tumor established by monitoring growth by palpation. When the tumor size had reached approximately $0.5 \mathrm{~cm}$ in diameter, the animals were randomized into three groups and therapy was initiated. Local injections of $250 \mu \mathrm{l}$ of TIMP-1-GPI at $14 \mathrm{ng} / \mathrm{ml}, 14 \mathrm{ng} / \mathrm{ml}$ of rhTIMP-1 protein or vehicle ( $1 \times$ phosphate-buffered saline, PBS, $0.025 \%$ Triton $\mathrm{X}-100 \mathrm{H})$ were applied. The injections were made every other day around and in the middle of the tumor (a total of five points, $50 \mu \mathrm{l}$ each) for a total of five treatments over a span of 10 days. At least three mice from each group were sacrificed at post-injection day 14 and tumors were isolated and measured. The tumor volume was determined by the following formula, $V=\pi \times$ length $\times$ width $\times$ height $/ 6$.

\section{Immunohistochemistry}

Resultant tumors were formaldehyde-fixed and embedded in paraffin wax. Then $3-\mu \mathrm{m}$ serial sections were generated. Tissues were deparaffinized in xylene and rehydrated in a graded series of ethanol. Endogenous peroxidase was blocked by incubation with $3 \%$ hydrogen peroxide $\left(\mathrm{H}_{2} \mathrm{O}_{2}\right)$. Antigen retrieval was performed in antigen retrieval solution (Dako, USA) in a microwave oven. Endogenous avidin and biotin were blocked using the Avidin/Biotin Blocking Kit (Vector, USA). The primary antibodies were diluted in PBS containing $3 \%$ bovine serum albumin (BSA). In addition, the slides were treated for $20 \mathrm{~min}$ with blocking solution ( $8 \%$ goat serum in PBS with $3 \%$ BSA) before the primary antibody was applied. The antibodies used included monoclonal rabbit anti-Ki67 antibody (ab16667, Abcam, USA), polyclonal rabbit antiCD31 antibody (ab28364, Abcam, USA). Overnight incubation with the primary antibodies was followed by incubation with the biotinylated secondary antibody (goat anti-rabbit, BA1000 , Vector, USA), and the ABC reagent for signal amplification (Vectastain ABC-Peroxidase Kits, PK-4000, Vector, USA). Between incubation steps, the slides were washed in TBS buffer. 3,3'-diaminobenzidine (DAB, Dako, USA) was used to develop color. Slides were counter-stained with hematoxylin, and mounted in Kaisers Glycerinegelatine (Merck,
Germany) and coverslips. After immunohistochemical staining against Ki67, slides were observed under high magnification scope $(\times 400)$. Areas showing the highest Ki67 density were chosen and photographs were taken. These photographs were analyzed by Image-J program (NIH, MD, USA). The Ki67 index were evaluated in a blinded manner and calculated as Ki67-positive cells divided by all tumor cells in one field.

\section{Statistics}

The results are presented as mean \pm SD for each sample. Statistical significance was assessed by comparing mean values using the $t$ test for random samples (IBM SPSS 19.0 for Windows). $p$ values $<0.05$ were considered significant.

\section{Results}

Enhanced incorporation of TIMP-1 into cell surface by fusion with a GPI anchor

Fluorescent staining showed incorporation of exogenously added TIMP-1-GPI into the surface membranes of HT1080 cells. HT1080 cells were incubated with $14 \mathrm{ng} / \mathrm{ml}$ of TIMP-1GPI or $14 \mathrm{ng} / \mathrm{ml}$ rhTIMP-1 control proteins for $2 \mathrm{~h}$. Surfaceassociated TIMP-1 protein was then detected using anti-TIMP1 monoclonal antibody and fluorescent staining. Control rhTIMP-1 did not change the fluorescent signal as compared with vehicle control group (Fig. 1a and c). However, GPIanchored TIMP-1 led to a strong surface signal for TIMP-1 (Fig. 1e). This was supported by fluorescence-activated cell sorting (FACS) analysis, which detected strong surface signal for TIMP-1 in TIMP-1-GPI treated group (Fig. 1f). However, addition of rhTIMP-1 or vehicle control did not lead to change in the FACS shift (Fig. 1b, d). Similar results were obtained for cells treated with $7 \mathrm{ng} / \mathrm{ml}$ TIMP-1-GPI (data not shown) confirming results from previous studies [23-26].

TIMP-1-GPI inhibits proliferation, clone formation and migration of HT1080 cells in vitro

Djafarzadeh et al. have previously shown that TIMP-1-GPI treatment can enhance or suppress cell viability/proliferation depending on the specific cell types studied [24-27, 34]. The effect of TIMP-1 surface engineering on HT1080 cells were determined using MTT assays. Exogenously added TIMP-1GPI protein was found to elicit a dose- and time-dependent inhibition in MTT activity of HT1080 in vitro (Fig. 2a). Subsequent studies were conducted using either 7 or $14 \mathrm{ng} / \mathrm{ml}$ TIMP-1-GPI depending upon the specific assay performed.

Two hundred vehicle, rhTIMP-1, or TIMP-1-GPI treated HT1080 cells were seeded in six-well plates. Fourteen days 
Fig. 1 TIMP-1-GPI

incorporation into cell

membranes of fibrosarcoma.

To demonstrate incorporation

of GPI-anchored TIMP-1 protein

into cell membranes, purified

TIMP-1-GPI, rhTIMP-1 or

vehicle control reagent was added to HT1080 cells. TIMP-1 was detected on the cell surface by fluorescent staining and FACS analysis. a, b Vehicle control group showed no surface signal for TIMP-1; c, d rhTIMP-1 treated cells showed no surface signal for TIMP-1; e, f TIMP-1GPI treatment lead to strong TIMP-1 signal on the cell surface (FACS graphs: gray histograms represent isotype control stain, solid red line histograms represent the anti-TIMP-1 antibody staining)
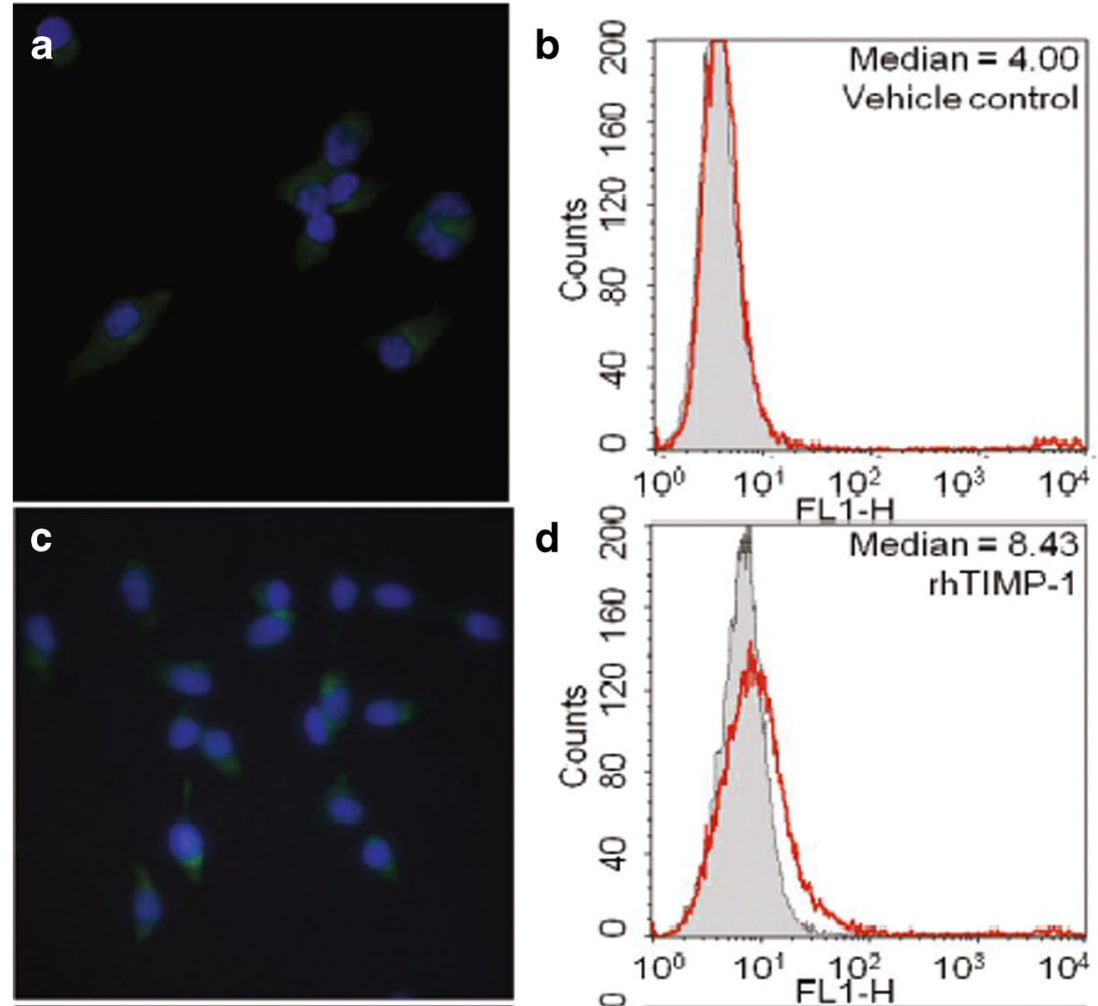

d
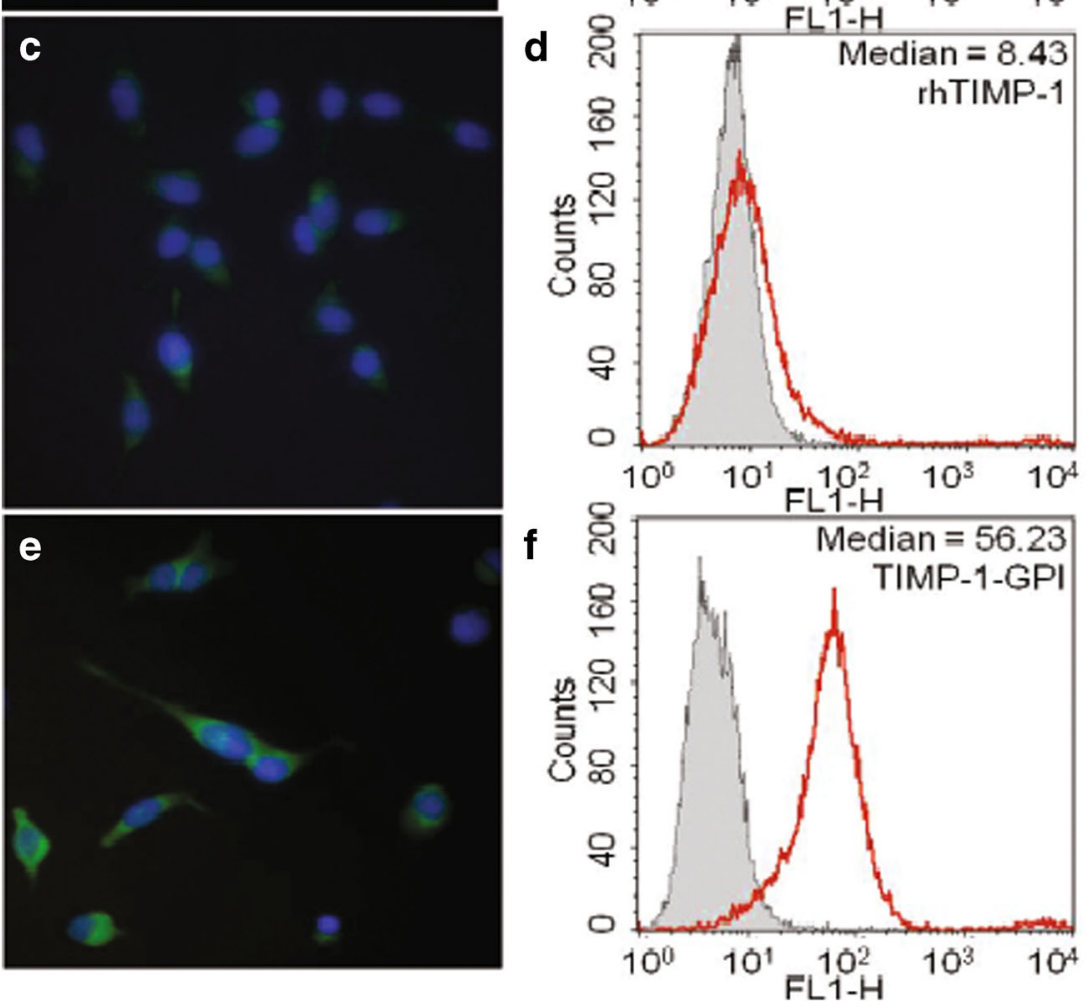

later, the clone formation ability of the cells was evaluated using crystal violet staining. As compared with the vehicle control or $7 \mathrm{ng} / \mathrm{ml}$ rhTIMP-1 group, $7 \mathrm{ng} / \mathrm{ml}$ TIMP-1-GPI significantly inhibited tumor cell clone formation in vitro (vehicle control vs. TIMP-1-GPI, $99.0 \pm 5.0$ vs. $13.0 \pm 1.7$ $\left[\right.$ mean \pm SD], ${ }^{*} p<0.01$; rhTIMP-1 vs. TIMP-1-GPI, $87.7 \pm$ 4.0 vs. $13.0 \pm 1.7, * * p<0.01$, Fig. 2 b).

The effect of TIMP-1-GPI vs. rhTIMP-1 treatment on the ability of HT1080 cells to migrate was further investigated using modified Boyden Chamber assays with induced migration to FBS. Treatment of the HT1080 cells with $7 \mathrm{ng} / \mathrm{ml}$ of TIMP-1-GPI led to a significant suppression of migration, while no effect was observed with rhTIMP-1 control protein (vehicle control vs. TIMP-1-GPI, $36.7 \pm 12.4$ vs. $13.4 \pm 2.0$, $* p=0.03$; rhTIMP-1 vs. TIMP-1-GPI, $34.3 \pm 3.7$ vs. $13.4 \pm 2.0$, $* * p=0.001$; Fig. 2c).
Treatment of HT1080 cells with TIMP-1-GPI enhances apoptosis

The influence of TIMP-1-GPI treatment on the apoptosis rate of fibrosarcoma cells was assessed using Annexin-V FACS assays. In the vehicle control group, the percentage of apoptotic cells was detected as $2.18 \pm 0.10 \%$. Treatment with rhTIMP-1 did not alter the percentage of apoptotic cells significantly $(3.08 \pm 1.18 \%, p=0.26)$. However, the apoptotic cell percentage increased to $5.13 \pm 1.29 \%$ after treatment with TIMP-1-GPI group, which was significantly increased as compared to the vehicle control group (* $*^{*}=0.017$; Fig. 3a).

To determine the effect of TIMP-1-GPI treatment on the susceptibility of fibrosarcoma to chemotherapy, doxorubicin, the chemotherapeutic agent of choice for fibrosarcoma patients 
a

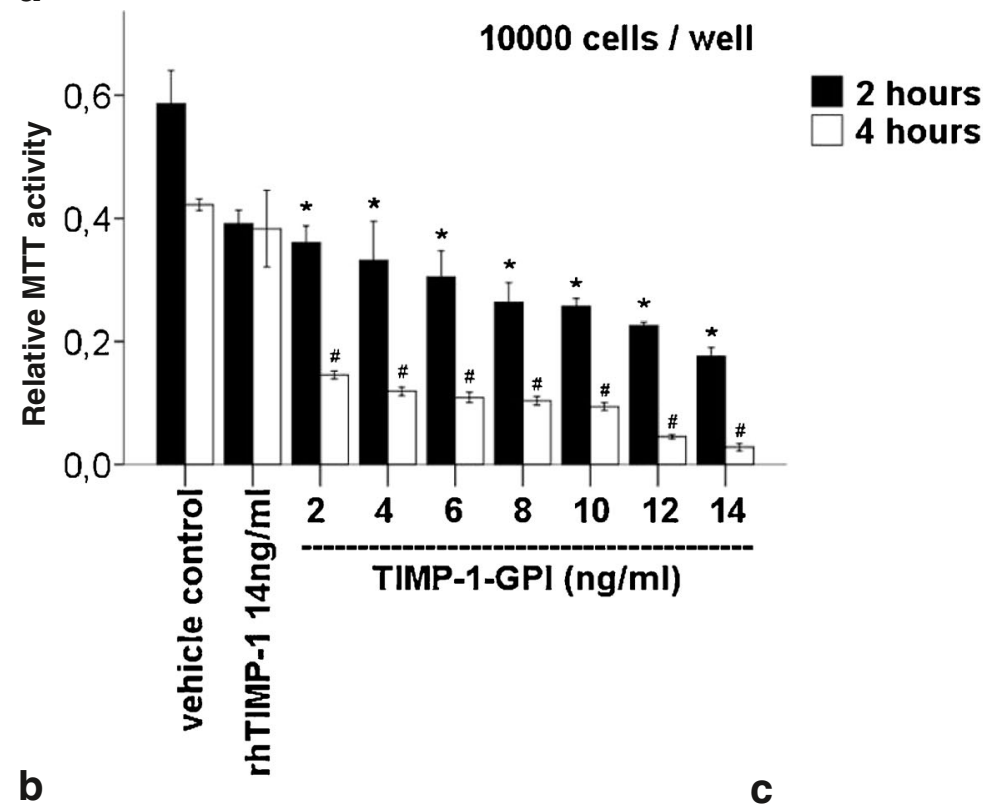

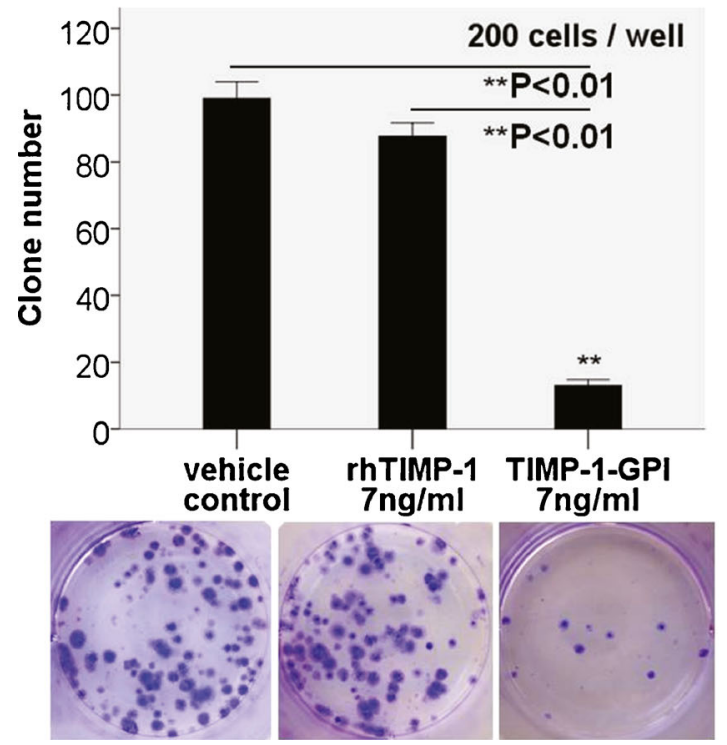

Fig. 2 GPI-anchored TIMP-1 suppresses proliferation, clone formation and migration of HT1080 cells in vitro. a The effect of increasing levels of TIMP-1-GPI $(2,4,6,8,10,12,14 \mathrm{ng} / \mathrm{ml})$ or rhTIMP-1 control protein $(14 \mathrm{ng} / \mathrm{ml})$ on the proliferation of HT1080 was measured using a commercial proliferation assay CCK-8 kit; $\mathbf{b}$ the effect of vehicle, rhTIMP-1

was added to the cell culture. In these studies, the therapy combining TIMP-1-GPI and doxorubicin showed a significantly higher percentage of apoptotic cells (TIMP-1-GPI+ doxorubicin: $8.35 \pm 0.57 \%$, vs. vehicle control: $2.03 \pm 0.00 \%$, ${ }^{* *} p=0.004$; vs. doxorubicin alone: $3.08 \pm 1.21 \%, * p=0.03$; Fig. 3b) as compared to the control groups of no treatment or treatment with doxorubicin alone. The rhTIMP-1 control did not affect susceptibility of fibrosarcoma cells to doxorubicin (TIMP-1-GPI+doxorubicin vs. rhTIMP-1+doxorubicin: $2.71 \pm 0.06 \%,{ }^{* *} p=0.005$; Fig. 3b).

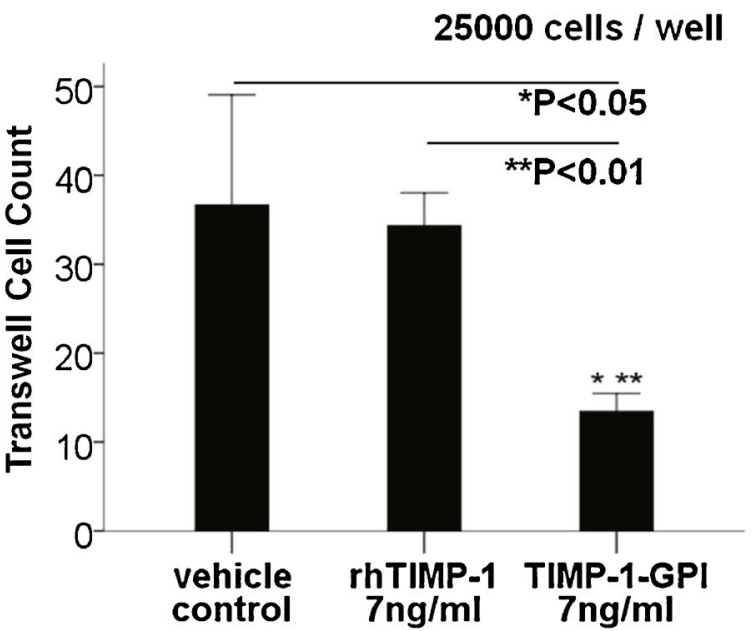

(7 ng/ml) and TIMP-1-GPI (7 ng/ml) on the clonogenicity of HT1080 was demonstrated using a clone formation assay. Exemplary pictures of each group are shown in the lower row; $\mathbf{c}$ the effect of vehicle, rhTIMP-1 ( $7 \mathrm{ng} / \mathrm{ml})$ or TIMP-1-GPI $(7 \mathrm{ng} / \mathrm{ml})$ on the migration of HT1080 was demonstrated by a migration assay

Effective inhibition of side population cells in HT1080 by TIMP-1-GPI treatment

The ability of cancer cell subpopulations to exclude Hoechst 33342 dye is used to identify stem cell-like SP. This assay was able to demonstrate a distinct side population in the HT1080 fibrosarcoma cells which generally accounted for $0.54 \% \pm 0.17 \%$ of HT1080 cells (mean \pm SD). While $7 \mathrm{ng} /$ $\mathrm{ml}$ TIMP-1-GPI treatment showed an effect (data not shown), $14 \mathrm{ng} / \mathrm{ml}$ TIMP-1-GPI led to a $55.6 \%$ reduction in the 


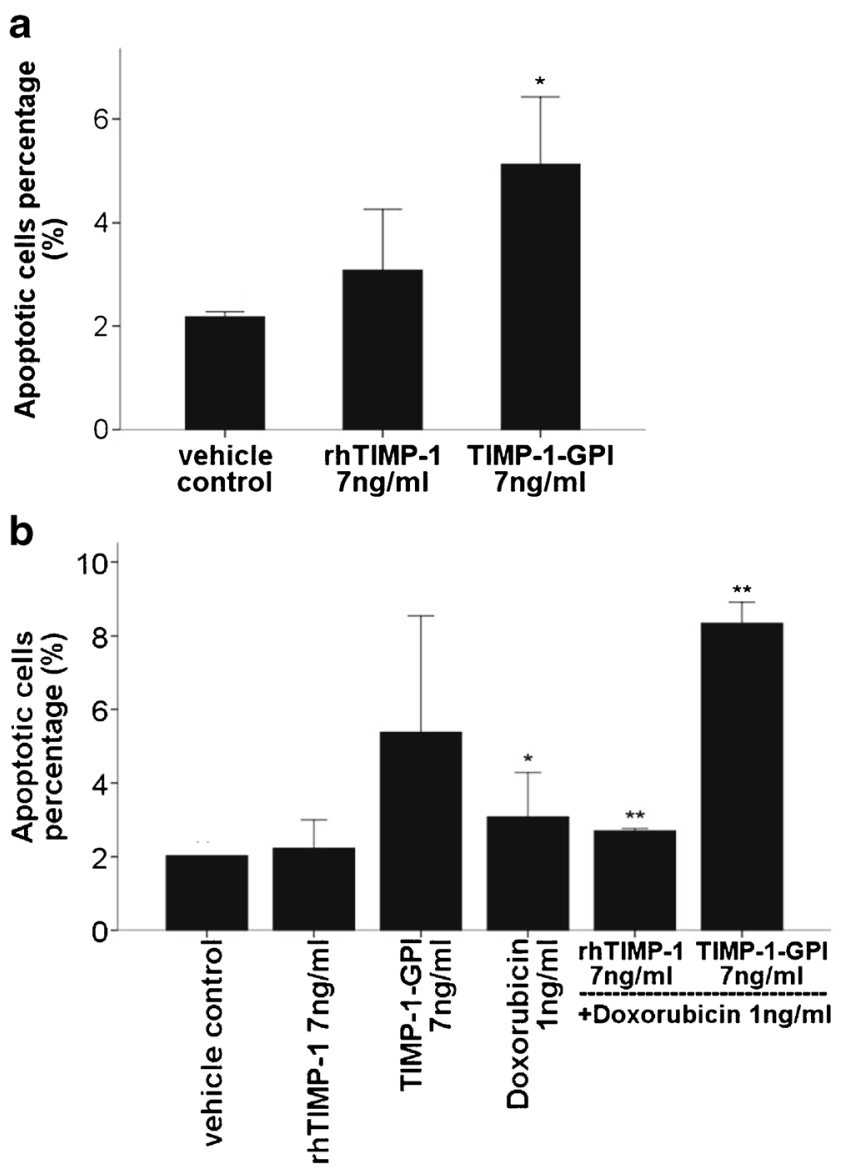

Fig. 3 TIMP-1-GPI induces apoptosis in HT1080 cells. a TIMP-1-GPI induced more than two-fold the presence of apoptotic cells as compared with control group, ${ }^{*} p<0.05$; b TIMP-1-GPI combined with the chemotherapeutic drug doxorubicin significantly increased apoptosis in cells as compared with vehicle control $(* * p<0.01)$, doxorubicin alone $(* p<0.05)$ or rhTIMP- $1+$ doxorubicin $(* * p<0.01)$ treatment group. This indicates a more than additive effect on apoptosis by combining TIMP-1-GPI and doxorubicin. Apoptotic cells are positive for both Annexin V and PI [37]. The experiments are representative of at least three replicates. The error bars indicate standard deviation of the mean

proportion of side population cells as compared to control vehicle treated HT1080 cells $(0.54 \% \pm 0.17 \%$ vs. $0.24 \% \pm 0.05 \%$; ${ }^{*} p=0.039$; Fig. 4 ).

TIMP-1-GPI inhibits tumor growth in vivo

Subcutaneous HT1080 tumor xenografts were established by dorsal injection of $1.0 \times 10^{6}$ HT1080 cells. When the s.c. tumors reached approximately $0.5 \mathrm{~cm}$ in diameter, the tumors were randomized and treatment with vehicle, rhTIMP-1 $(14 \mathrm{ng} / \mathrm{ml})$ and TIMP-1-GPI $(14 \mathrm{ng} / \mathrm{ml})$ was initiated. The samples were injected locally and in equal distribution at four points surrounding the tumor and into the center of the tumor.

Treatment with TIMP-1-GPI lead to a significant inhibition in tumor growth as compared to vehicle control and rhTIMP-1 treatment groups (vehicle control vs. TIMP-1-GPI: $151.9 \pm 54.8 \mathrm{~cm}^{3}$ vs. $22.0 \pm 25.9 \mathrm{~cm}^{3},{ }^{*} p=0.021$; rhTIMP-1 vs.
TIMP-1-GPI: $107.9 \pm 29.8 \mathrm{~cm}^{3}$ vs. $22.0 \pm 25.9 \mathrm{~cm}^{3}, * * p=0.020$; Fig. 5). No significant difference were seen between the vehicle control group and the group treated with rhTIMP-1 $(p=0.29)$.

Ex vivo analysis shows that TIMP-1-GPI inhibits tumor proliferation and angiogenesis in vivo

In vitro assays had demonstrated that TIMP-1-GPI treatment inhibits HT1080 proliferation in a dose- and time-dependent manner. This was evaluated ex vivo on tissue samples from the murine xeno tumor model using immunohistochemistry. Tissue sections were stained with anti-Ki67 and anti-CD31 antibodies to assess effects of TIMP-1-GPI on tumor proliferation and angiogenesis ex vivo.

The Ki67 index (Ki67 / total cells) was used to assess the general proliferation rate of cells within the tissue samples. As compared to the vehicle control and rhTIMP-1 group, the Ki67 proliferation index was significantly decreased in the TIMP-1-GPI treatment group (vehicle control vs. TIMP-1-GPI: $0.55 \pm 0.12$ vs. $0.20 \pm 0.05,{ }^{*} p=0.01$; rhTIMP-1 vs. TIMP-1-GPI: $0.61 \pm 0.09$ vs. $0.20 \pm 0.05, * * p=0.002$; Fig. $6 \mathrm{a}, \mathrm{b}$ ). There was no significant difference seen between the vehicle control and rhTIMP-1 groups $(p=0.304)$. Exemplary examples of each anti-Ki67 stain are provided in Fig. 6 b.

Anti-CD31 staining was used to evaluate the microvessel density of each treatment group. Although there was no statistical difference demonstrated between these groups with regards to microvessel density (Supplement Table. 1), assessment of the TIMP-1-GPI treated tumors suggested a trend towards decreased CD31 positive signals (black arrowheads, upper row of Fig. 6a).

\section{Discussion}

In vivo, the balance between MMPs and TIMPs helps to determine whether matrix resorption or deposition occurs [35]. During cancer progression, this balance is shifted towards the over-expression of proteases. However, broad-spectrum systemic inhibition of MMPs, e.g., with Marimastat $(\mathcal{C}$ have failed to show effectiveness in clinical trials for various reasons [36].

While the TIMP proteins inhibit MMP activity, and thus, are capable of modulating tumor growth and metastasis, they are also multifunctional proteins that modulate cell viability, proliferation, apoptosis, and angiogenesis. The TIMP family members have been reported to have conflicting actions on cancer growth [37]. Treatment with native TIMP-1 can produce both tumor promoting and inhibiting results. This appears to vary with the cancer entity examined, the local concentration of the protein, status of malignant tumor growth used, and the target organ for metastasis. In general, high levels of TIMP-1 appear to be necessary for effective 

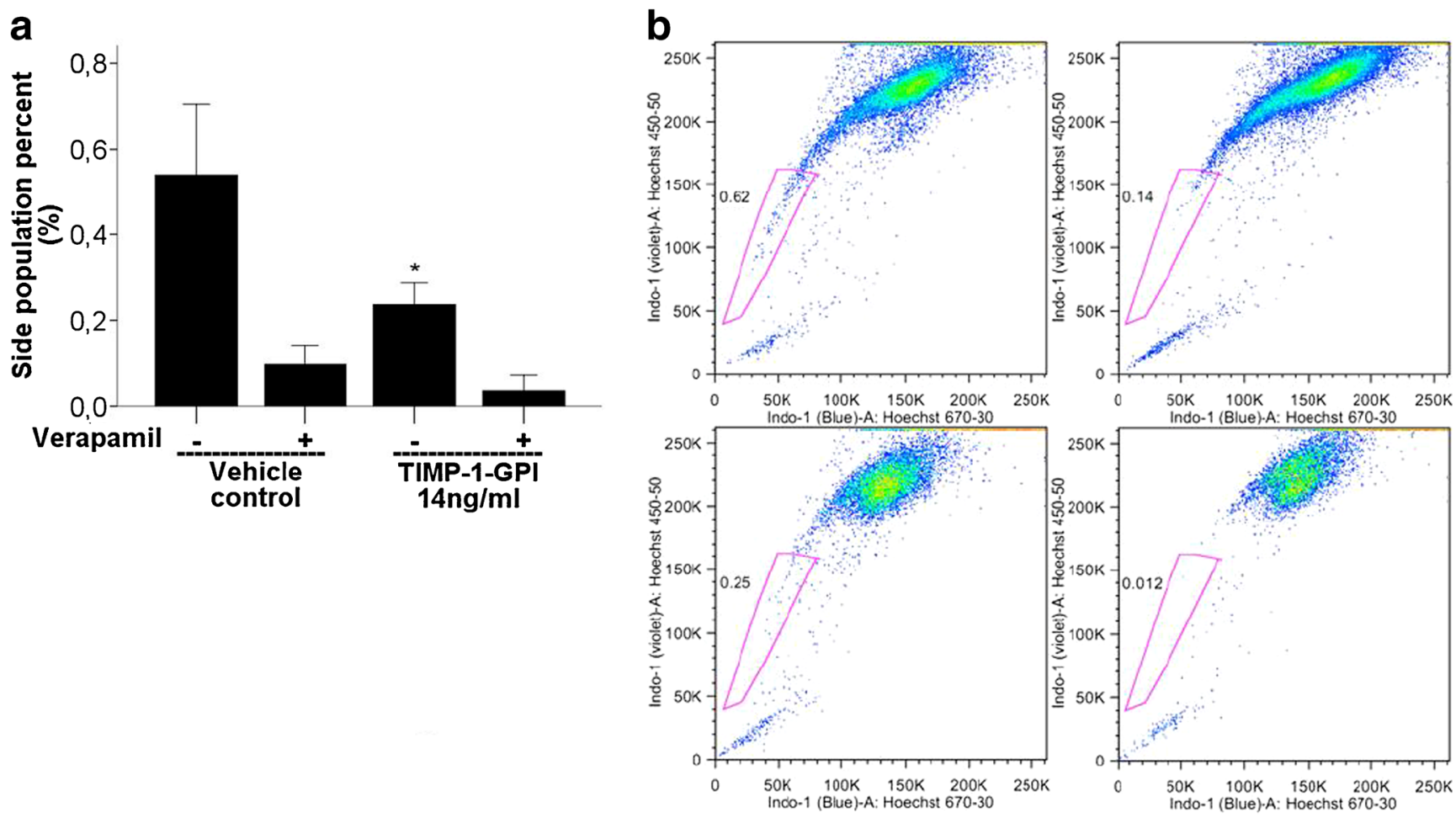

Fig. 4 TIMP-1-GPI inhibits side population cells. a TIMP-1-GPI treatment led to significant reduction in the level of side population cells as compared to the vehicle control group ( 0.54 vs. $0.24 \% * p=0.039)$. As a calcium channel blocker, verapamil abrogates the dye efflux in almost all cells and serves as control in this experiment. b Exemplary side population FACS analysis of each group is shown (clockwise from upper left: vehicle control -verapamil; Vehicle control +verapamil; TIMP-1-GPI $14 \mathrm{ng} / \mathrm{ml}$ +verapamil; TIMP-1-GPI $14 \mathrm{ng} / \mathrm{ml}$-verapamil) inhibition of tumor growth, whereas lower systemic levels of TIMP-1 can promote metastases [38]. We have previously

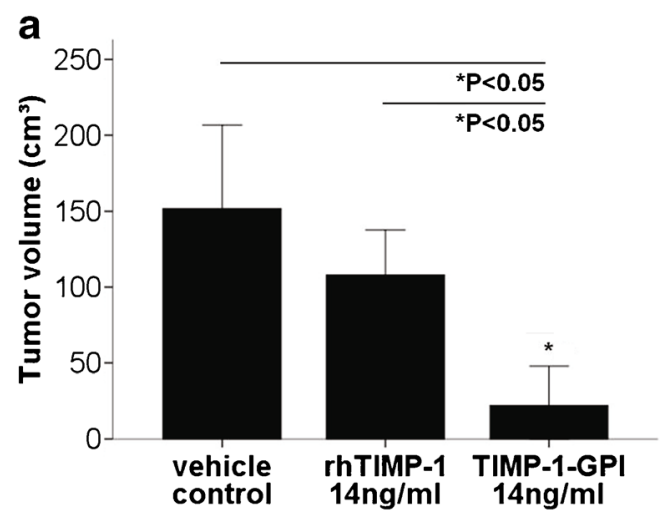

b

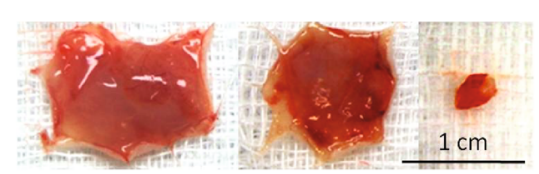

Fig. 5 Effect of locally applied TIMP-1-GPI on tumor growth in vivo. a Local injection of TIMP-1-GPI $(250 \mu \mathrm{l}$ of $14 \mathrm{ng} / \mathrm{ml})$ significantly inhibited growth of xenografted HT1080 tumors as compared to rhTIMP-1 (250 $\mu$ l of $14 \mathrm{ng} / \mathrm{ml}$ ) or vehicle control. Injections were made every other day around and in the middle of the tumor (a total of five points, $50 \mu \mathrm{l} \mathrm{each)} \mathrm{for}$ a total of 5 treatments over a span of 10 days; $\mathbf{b}$ Exemplary photograph of subcutaneous tumor xenografts at post-injection day 14 demonstrated that treatment with TIMP-1-GPI, which leads to high local expression only on the cell surface, results in dramatic changes in biological activity of the protein. This is associated with changes in the surface stoichiometry of the proteins - relative to that seen with the native protein.

TIMP-1 is a broadly acting soluble inhibitor that can sometimes be detected on the cell surface through its association with surface-bound proteins [39-41]. Focusing TIMP-1 to the plasma membrane via a GPI anchor redirects this inhibitor to the caveola-enriched lipid raft compartment [42]. Exogenously administered TIMP-1-GPI was shown here to efficiently insert into the cell membranes of HT1080 cells (Fig. 1) resulting in inhibition of proliferation, reduced clone formation and migration, enhanced apoptosis, and reduction in the level of side population cells.

Although cancer cells can express a broad range of MMPs, the stromal compartment also responds and displays active expression of MMPs, e.g., by fibroblasts or endothelial cells [43]. Therefore, effective cancer treatments based on this biology should address both the cancer cells and the surrounding stromal tissue [44]. Local application of TIMP-1-GPI will equally influence cancer and stromal compartments and therefore meets this therapeutic criterion. In this regard, we have recently shown that TIMP-1-GPI treatment is not deleterious to normal tissue, and can even show benefit in in vitro and in vivo models of wound healing [37]. 


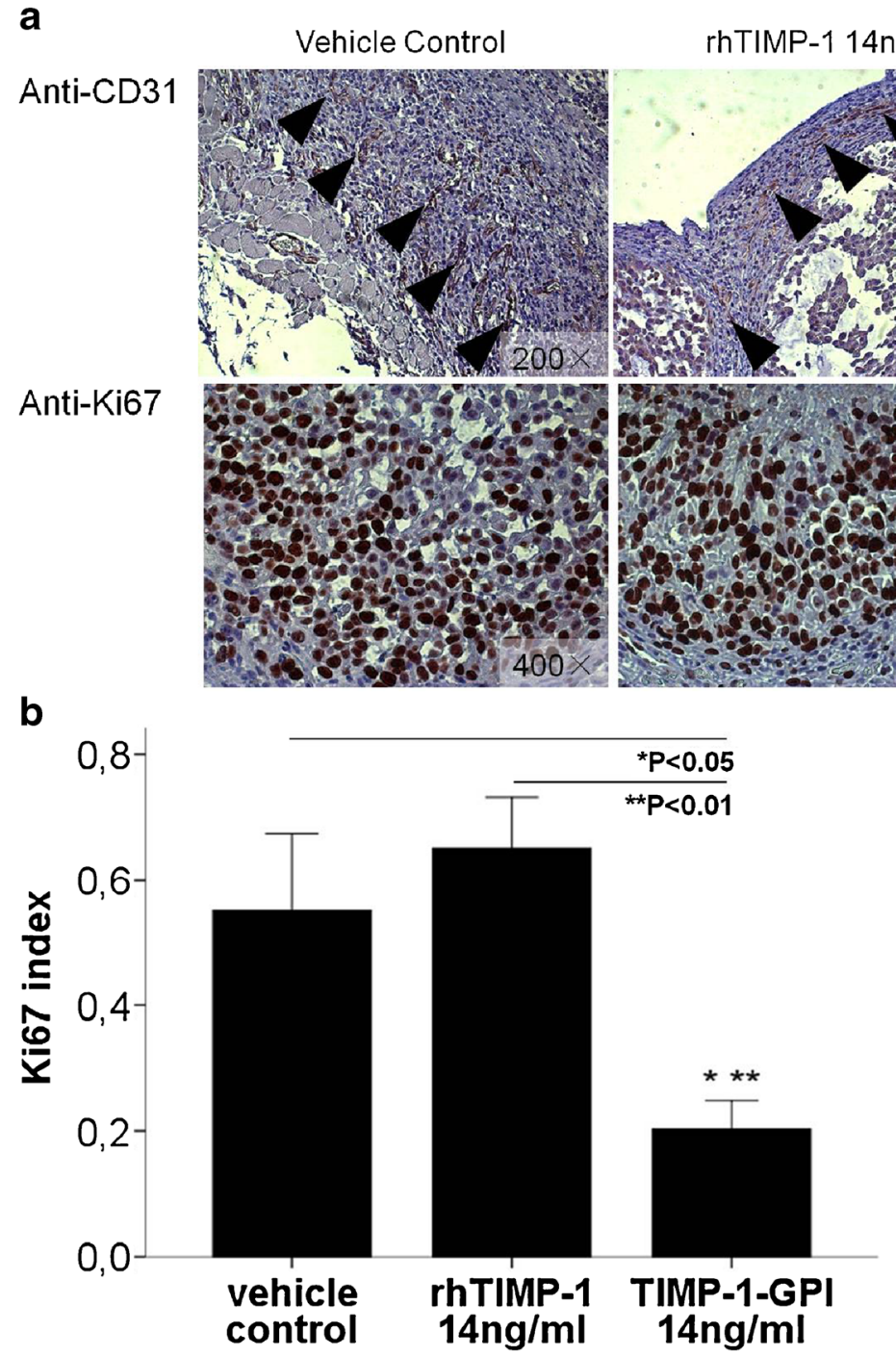

Fig. 6 Effect of locally applied TIMP-1-GPI on tumor proliferation and angiogenesis in vivo. a Exemplary pictures of anti-CD31 and anti-Ki67 antibodies staining. CD31 (black arrowheads) and Ki67 positive signals are less in TIMP-1-GPI treated group; b Ki67 index decreased significantly

after TIMP-1-GPI treatment compared with vehicle control and rhTIMP-1 groups (vehicle control vs. TIMP-1-GPI, ${ }^{*} p=0.01$; rhTIMP-1 vs. TIMP-1GPI, ${ }^{* *} p=0.002$ )

The anti-tumor efficacy of this fusion protein was validated in vivo using a subcutaneous xenograft model. Injection of TIMP-1-GPI protein suppressed experimental fibrosarcoma cell proliferation and decreased overall tumor growth in vivo with no deleterious effects on normal tissue (Fig. 5). Related anti-tumor effects of TIMP-1-GPI treatment were previously described in experimental colorectal cancer as well as in melanoma and are supportive of our findings $[24,34]$.

Local recurrence of sarcoma after surgical resection represents a major challenge in treating this tumor entity. Cancer cells have evolved various mechanisms to evade chemotherapy including reduced sensitivity to apoptosis [45]. Treatment of renal cell carcinoma cells with TIMP-1-GPI was previously shown to increase sensitivity to FAS-mediated apoptosis. In melanoma, similar increments in sensitivity to apoptosis were achieved by TIMP-1-GPI treatment when combined with hyperthermia [24]. In the data presented here, treatment of fibrosarcoma cells with TIMP-1-GPI also led to enhanced apoptosis. Hyperthermia, a frequently used treatment modality in sarcoma, might increase the effectiveness of TIMP-1GPI to induce sensitivity to apoptosis even further and it therefore may enhance chemotherapeutic effectiveness.

In various human cancer cell lines, treatment with high concentrations of recombinant TIMP-1, or artificial overexpression of TIMP-1 reduced susceptibility of the cells to chemotherapy induced apoptosis $[46,47]$. Similar results were seen here in that treatment of HT1080 with low levels of rhTIMP-1 led to a slightly lower, but statistically not significant response to doxorubicin. By contrast, treatment with nanogram quantities of TIMP-1-GPI lead to enhanced sensitivity of the HT1080 cells to doxorubicin. 
The concept of CSC ascribes the cancer properties of tumor initiation, progression, metastasis, recurrence and chemoresistance to a subset of cancer cells that have been termed CSCs. These cells have the capability to undergo selfrenewal, unlimited proliferation and multi-potentiality. SP cancer cells are isolated by their ability to exclude Hoechst33342-dye via $\mathrm{ABC}$ transporters, and are proposed to represent CSCs in diverse tumor entities [48, 49]. It is thought that long-term progression free survival in cancer patients will require effective targeting of CSCs to eliminate these largely therapy resistant "seed" populations. The SP subpopulation identified in the human fibrosarcoma cell line HT1080 was effectively reduced by treatment with TIMP-1-GPI.

Patients suffering from soft tissue sarcoma frequently undergo repeated resections of the tumor due to recurrences. In these settings, intraoperative peritumoral application of the therapeutic fusion protein TIMP-1-GPI as an adjuvant to surgery could help maintain tumor control by targeting microscopic residual tumor cells in the context of resection. The direct injection of TIMP-1-GPI could help minimize tumor growth in non-resectable tumors. Treatment with TIMP-1GPI may contribute to the establishment of novel therapeutic strategies targeting cancer stem cell.

Acknowledgments We thank Monika Hofstetter (Clinical Biochemistry Group, Medizinische Klinik und Poliklinik IV, University of Munich, Germany) for assistance in purification of recombinant proteins.

Conflict of Interest All funding for the research described here was provided through granting agencies of the German government or the University of Munich. The work was supported by grants from the Deutsche Forschungsgemeinschaft (DFG BR 1614/7-1 CJB; DFG SPP1190 "Tumor Vessel Interface" to CJB and PJN; DFG NE 648/2-4 to PJN) and the Förderprogram für Forschung und Lehre (HN) of the University of Munich. No conflict of Interest exists with these agencies. Various patents covering the general class of proteins described here are held by PJN.

Open Access This article is distributed under the terms of the Creative Commons Attribution License which permits any use, distribution, and reproduction in any medium, provided the original author(s) and the source are credited.

\section{References}

1. Overall CM, Kleifeld O (2006) Tumour microenvironment—opinion: validating matrix metalloproteinases as drug targets and anti-targets for cancer therapy. Nat Rev Cancer 6:227-239

2. Coussens LM, Fingleton B, Matrisian LM (2002) Matrix metalloproteinase inhibitors and cancer: trials and tribulations. Science 295:23872392

3. Kruger A, Kates RE, Edwards DR (2010) Avoiding spam in the proteolytic internet: future strategies for anti-metastatic MMP inhibition. Biochim Biophys Acta 1803:95-102

4. Ward RV, Hembry RM, Reynolds JJ, Murphy G (1991) The purification of tissue inhibitor of metalloproteinases- 2 from its $72 \mathrm{kDa}$ progelatinase complex. Demonstration of the biochemical similarities of tissue inhibitor of metalloproteinases-2 and tissue inhibitor of metalloproteinases-1. Biochem J 278(Pt 1):179-187

5. Howard EW, Bullen EC, Banda MJ (1991) Preferential inhibition of 72- and $92-\mathrm{kDa}$ gelatinases by tissue inhibitor of metalloproteinases2. J Biol Chem 266:13070-13075

6. Quantin B, Murphy G, Breathnach R (1989) Pump-1 cDNA codes for a protein with characteristics similar to those of classical collagenase family members. Biochemistry 28:5327-5334

7. Knauper V, Osthues A, DeClerck YA, Langley KE, Blaser J et al (1993) Fragmentation of human polymorphonuclear-leucocyte collagenase. Biochem J 291(Pt 3):847-854

8. Murphy G, Segain JP, O’Shea M, Cockett M, Ioannou C et al (1993) The $28-\mathrm{kDa}$ N-terminal domain of mouse stromelysin-3 has the general properties of a weak metalloproteinase. J Biol Chem 268:1543515441

9. Cornelius LA, Nehring LC, Harding E, Bolanowski M, Welgus HG et al (1998) Matrix metalloproteinases generate angiostatin: effects on neovascularization. J Immunol 161:6845-6852

10. Knauper V, Lopez-Otin C, Smith B, Knight G, Murphy G (1996) Biochemical characterization of human collagenase-3. J Biol Chem 271:1544-1550

11. English WR, Puente XS, Freije JM, Knauper V, Amour A et al (2000) Membrane type 4 matrix metalloproteinase (MMP17) has tumor necrosis factor-alpha convertase activity but does not activate proMMP2. J Biol Chem 275:14046-14055

12. Stracke JO, Hutton M, Stewart M, Pendas AM, Smith B et al (2000) Biochemical characterization of the catalytic domain of human matrix metalloproteinase 19. Evidence for a role as a potent basement membrane degrading enzyme. J Biol Chem 275:1480914816

13. English WR, Velasco G, Stracke JO, Knauper V, Murphy G (2001) Catalytic activities of membrane-type 6 matrix metalloproteinase (MMP25). FEBS Lett 491:137-142

14. Uria JA, Lopez-Otin C (2000) Matrilysin-2, a new matrix metalloproteinase expressed in human tumors and showing the minimal domain organization required for secretion, latency, and activity. Cancer Res 60:4745-4751

15. Khokha R, Waterhouse P, Yagel S, Lala PK, Overall CM et al (1989) Antisense RNA induced reduction in murine TIMP levels confers oncogenicity on Swiss 3t3-Cells. Science 243:947-950

16. Declerck YA, Perez N, Shimada H, Boone TC, Langley KE et al (1992) Inhibition of invasion and metastasis in cells transfected with an inhibitor of metalloproteinases. Cancer Res 52:701-708

17. Alonso DF, Skilton G, De Lorenzo MS, Scursoni AM, Yoshiji H et al (1998) Histopathological findings in a highly invasive mouse mammary carcinoma transfected with human tissue inhibitor of metalloproteinases-1. Oncol Rep 5:1083-1087

18. Hayakawa T, Yamashita K, Ohuchi E, Shinagawa A (1994) Cell growth-promoting activity of tissue inhibitor of metalloproteinases2 (TIMP-2). J Cell Sci 107(Pt 9):2373-2379

19. Hayakawa T, Yamashita K, Tanzawa K, Uchijima E, Iwata K (1992) Growth-promoting activity of tissue inhibitor of metalloproteinases-1 (TIMP-1) for a wide range of cells. A possible new growth factor in serum. FEBS Lett 298:29-32

20. Yoshiji H, Harris SR, Raso E, Gomez DE, Lindsay CK et al (1998) Mammary carcinoma cells over-expressing tissue inhibitor of metalloproteinases-1 show enhanced vascular endothelial growth factor expression. Int J Cancer 75:81-87

21. Goss KJ, Brown PD, Matrisian LM (1998) Differing effects of endogenous and synthetic inhibitors of metalloproteinases on intestinal tumorigenesis. Int J Cancer 78:629-635

22. Kruger A, Fata JE, Khokha R (1997) Altered tumor growth and metastasis of a T-cell lymphoma in Timp-1 transgenic mice. Blood 90:1993-2000

23. Medof ME, Nagarajan S, Tykocinski ML (1996) Cell-surface engineering with GPI-anchored proteins. FASEB J 10:574-586 
24. Djafarzadeh R, Milani V, Rieth N, von Luettichau I, Skrablin PS et al (2009) TIMP-1-GPI in combination with hyperthermic treatment of melanoma increases sensitivity to FAS-mediated apoptosis. Cancer Immunol Immunother 58:361-371

25. Djafarzadeh R, Mojaat A, Vicente AB, von Luttichau I, Nelson PJ (2004) Exogenously added GPI-anchored tissue inhibitor of matrix metalloproteinase-1 (TIMP-1) displays enhanced and novel biological activities. Biol Chem 385:655-663

26. Djafarzadeh R, Noessner E, Engelmann H, Schendel DJ, Notohamiprodjo M et al (2006) GPI-anchored TIMP-1 treatment renders renal cell carcinoma sensitive to FAS-meditated killing. Oncogene 25:1496-1508

27. Djafarzadeh R, Sauter M, Notohamiprodjo S, Noessner E, Goyal P et al (2012) Recombinant GPI-anchored TIMP-1 stimulates growth and migration of peritoneal mesothelial cells. PLoS One 7:e33963

28. Schmuck R, Warneke V, Behrens HM, Simon E, Weichert W et al (2011) Genotypic and phenotypic characterization of side population of gastric cancer cell lines. Am J Pathol 178:1792-1804

29. Marquardt JU, Raggi C, Andersen JB, Seo D, Avital I et al (2011) Human hepatic cancer stem cells are characterized by common stemness traits and diverse oncogenic pathways. Hepatology 54:1031-1042

30. Wang CYY, Wei QX, Han I, Sato S, Ghanbari-Azarnier R et al (2012) Hedgehog and notch signaling regulate self-renewal of undifferentiated pleomorphic sarcomas. Cancer Res 72:1013-1022

31. Rasheed S, Nelson-Rees WA, Toth EM, Arnstein P, Gardner MB (1974) Characterization of a newly derived human sarcoma cell line (HT-1080). Cancer 33:1027-1033

32. Kirby AC, Hill V, Olsen I, Porter SR (1995) LFA-3 delta D2: a novel in vivo isoform of lymphocyte function-associated antigen 3 . Biochem Biophys Res Commun 214:200-205

33. Mack M, Riethmuller G, Kufer P (1995) A small bispecific antibody construct expressed as a functional single-chain molecule with high tumor cell cytotoxicity. Proc Natl Acad Sci U S A 92:7021-7025

34. Raggi MC, Djafarzadeh R, Muenchmeier N, Hofstetter M, Jahn B et al (2009) Peritumoral administration of GPI-anchored TIMP-1 inhibits colon carcinoma growth in Rag-2 gamma chain-deficient mice. Biol Chem 390:893-897

35. Nagase H, Woessner JF Jr (1999) Matrix metalloproteinases. J Biol Chem 274:21491-21494

36. Hu J, Van den Steen PE, Sang QX, Opdenakker G (2007) Matrix metalloproteinase inhibitors as therapy for inflammatory and vascular diseases. Nat Rev Drug Discov 6:480-498
37. Egeblad M, Werb Z (2002) New functions for the matrix metalloproteinases in cancer progression. Nat Rev Cancer 2:161174

38. Kopitz C, Gerg M, Bandapalli OR, Ister D, Pennington CJ et al (2007) Tissue inhibitor of metalloproteinases-1 promotes liver metastasis by induction of hepatocyte growth factor signaling. Cancer Res 67:8615-8623

39. Brew K, Dinakarpandian D, Nagase H (2000) Tissue inhibitors of metalloproteinases: evolution, structure and function. Biochim Biophys Acta 1477:267-283

40. Klier CM, Nelson EL, Cohen CD, Horuk R, Schlondorff D et al (2001) Chemokine-induced secretion of gelatinase B in primary human monocytes. Biol Chem 382:1405-1410

41. Bode W, Maskos K (2003) Structural basis of the matrix metalloproteinases and their physiological inhibitors, the tissue inhibitors of metalloproteinases. Biol Chem 384:863-872

42. Sohail A, Sun Q, Zhao H, Bernardo MM, Cho JA et al (2008) MT4(MMP17) and MT6-MMP (MMP25), a unique set of membraneanchored matrix metalloproteinases: properties and expression in cancer. Cancer Metastasis Rev 27:289-302

43. Liotta LA, Kohn EC (2001) The microenvironment of the tumourhost interface. Nature 411:375-379

44. Bandapalli OR, Paul E, Schirmacher P, Brand K (2012) Opposite effects of tissue inhibitor of metalloproteinases-1 (TIMP-1) overexpression and knockdown on colorectal liver metastases. BMC Res Notes 5:14

45. Dunn GP, Old LJ, Schreiber RD (2004) The three Es of cancer immunoediting. Annu Rev Immunol 22:329-360

46. Guedez L, Stetler-Stevenson WG, Wolff L, Wang J, Fukushima P et al (1998) In vitro suppression of programmed cell death of B cells by tissue inhibitor of metalloproteinases-1. J Clin Invest 102:20022010

47. Davidsen ML, Wurtz SO, Romer MU, Sorensen NM, Johansen SK et al (2006) TIMP-1 gene deficiency increases tumour cell sensitivity to chemotherapy-induced apoptosis. Br J Cancer 95:11141120

48. Chiba T, Kita K, Zheng YW, Yokosuka O, Saisho H et al (2006) Side population purified from hepatocellular carcinoma cells harbors cancer stem cell-like properties. Hepatology 44:240-251

49. Haraguchi N, Utsunomiya T, Inoue H, Tanaka F, Mimori K et al (2006) Characterization of a side population of cancer cells from human gastrointestinal system. Stem Cells 24:506-513 